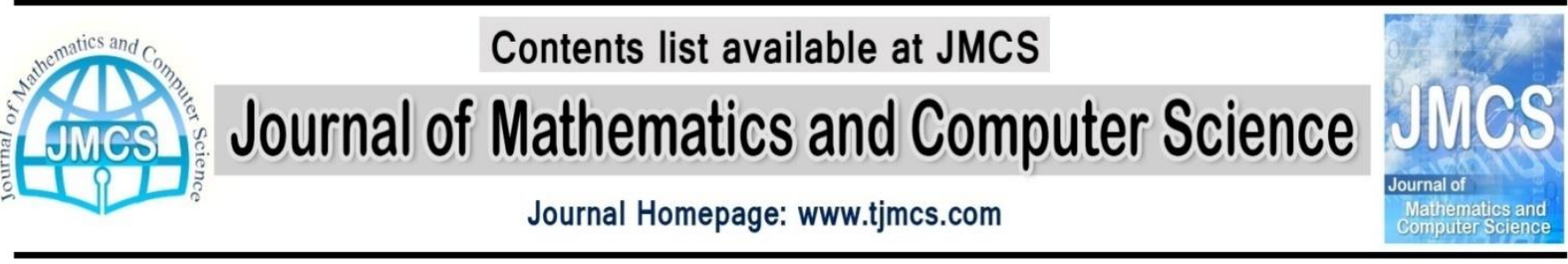

\section{On Approximate Solution of Fractional Order Logistic Equations by Operational Matrices of Bernstein Polynomials}

\author{
Hasib Khan 1,2,", Mohsen Alipour ${ }^{3}$, Rahmat Ali Khan', Haleh Tajadodi', Aziz Khan ${ }^{1}$ \\ ${ }^{1}$ Department of Mathematics, University of Malakand, Dir Lower, P. O Box 18000, Khybar \\ Pakhtunkhwa, Pakistan \\ ${ }^{2}$ Shaheed Benazir Bhutto University, Sheringal, Dir Upper, P. O Box 18000, Khybar \\ Pakhtunkhwa, Pakistan \\ ${ }^{3}$ Department of Mathematics, Faculty of Basic Science, Babol University of Technology, \\ P.O. Box 47148-71167, Babol, Iran. \\ ${ }^{4}$ Department of Mathematics, University of Mazandaran, P. O Box 47416-95447, Babolsar, \\ Iran. \\ *hasibkhan13@yahoo.com
}

\begin{abstract}
Article history:
Received November 2014

Accepted January 2015

Available online January 2015
\end{abstract}

\begin{abstract}
In this Paper we give a scheme for the numerical solution of fractional order Logistic equations (FOLE) using operational matrices for fractional order integration and multiplications based on Bernstein Polynomials (BPs). By this method we get the FOLE in the form of a system of algebraic equations which is simple in handling for the numerical solutions and better approximations are obtained. For the illustration of the efficiency and simplicity of the scheme, three examples are added in the paper.

Keywords: Fractional order Logistic equations, Bernstein Polynomials, Operational Matrices.
\end{abstract}

\section{Introduction}

The applications of fractional calculus can be studied in many scientific disciplines based on mathematical modeling including physics, aerodynamics, chemistry, signal and image processing, economics, electrodynamics, polymer rheology, economics, blood flow phenomena, biophysics, control theory and many others. For this, we recommend to [1-5]. Due to the wide range applications of fractional calculus and fractional dynamics, this area caught the interest of many researchers recently in [6-10].

Numerical solutions of fractional order differential equations have considered by many researchers such as in [12], H. Jafari et.al studied Abel differential equation of fractional order using Homotopy analysis 
method. In [16], D. Baleanu et.al consider fractional quadratic Riccati differential equations with the Riemann-Liouville derivative via the operational matrices of BPs. In [17], M. Alipour and D.Rosatamy used BPs for solving Abel's integral equation. In [18], M. Alipour and D. Rostamy consider the numerical solution of time varying fractional optimal control problem via operational matrices of BPs.

In the literature we see some papers on the study of FOLE including [20] by El-Sayed et al. In [19] S. Bhalekar and V. D. Gejji considered FOLE by the new iterative method introduced by D. Gejji and H. Jafari in [21].

Our interest in this paper is to produce a numerical scheme for the solution of FOLE using operational matrices of fractional order integration and multiplication of BPs

$$
D^{\alpha} x(t)=\beta x(t)(1-x(t)), t>0, \beta>0,
$$

With initial condition

$$
x(0)=b
$$

and $\alpha \in[0,1]$. In the following eq. (3), $D^{\alpha}$ is the Caputo fractional derivative [17]:

$$
D^{\alpha} f(x)=\left\{\begin{array}{cc}
\frac{1}{\Gamma(n-\alpha)} \int_{0}^{x} \frac{f(t) d t}{(x-t)^{1+\alpha-n}}, & n-1<\alpha<n, \\
\frac{d^{n}}{d x^{n}} f(x), \alpha=n &
\end{array}\right.
$$

We give some properties of fractional derivative and integral from the available resources in [3-5]:

$$
\begin{aligned}
& D_{x}^{\alpha} C=0,(\mathrm{C} \text { is a constant }) \\
& D_{x}^{\alpha} x^{\mu}(t)=\left\{\begin{array}{l}
0 \quad \beta \in N, \quad \mu<\lceil\alpha\rceil \\
\frac{\Gamma(\mu+1)}{\Gamma(\mu+1-\mu)}, \mu \in N, \mu \geq\lceil\alpha\rceil \text { or } \mu \notin N, \mu>\lfloor\alpha\rfloor,
\end{array}\right. \\
& I_{x}^{\alpha} D_{x}^{\alpha} f(x)=f(x)-\sum_{k=0}^{n-1} f^{(k)}(0) \frac{x^{k}}{k !}, \quad n-1<\alpha \leq n,
\end{aligned}
$$

Where $I_{x}^{\alpha}$ is the fractional Riemann-Liouville integral, given by

$$
I_{x}^{\alpha} f(x)=\frac{1}{\Gamma(\alpha)} \int_{0}^{x} \frac{f(t) d t}{(x-t)^{1-\alpha}}, \quad \alpha>0 .
$$

Organization of our Paper: In Section 2, we present BPs and approximation of functions via BPs. In section 3, we describe about the operational matrices of BPs. In section 4, we develop a numerical scheme for the FOLE (1). In section 5, Illustrative examples are given.

\section{Preliminary Results}

Recall that in [8], the BPs of mth degree defined on the interval $[0,1]$, given as under

$$
X_{i, m}(x)=\left(\begin{array}{c}
m \\
i
\end{array}\right) x^{i}(1-x)^{m-i}, 0 \leq i \leq m
$$

satisfy the recursive relation: 


$$
\mathrm{X}_{i, m}(x)=(1-x) \mathrm{X}_{i, m-1}(x)+x \mathrm{X}_{i-1, m-1}(x), \quad i=0,1, \ldots, m
$$

Using the Bionomial expension $(1-x)^{m-i}$, the BPs can be written in terms of linear combination of the basis function as follows

$$
\mathrm{X}_{i, m}(x)=\left(\begin{array}{c}
m \\
i
\end{array}\right) x^{i}(1-x)^{m-i}=\left(\begin{array}{c}
m \\
i
\end{array}\right) x^{i}\left(\sum_{k=0}^{m-i}(-1)^{k}\left(\begin{array}{c}
m-i \\
k
\end{array}\right) x^{k}\right)=\sum_{k=0}^{m-i}(-1)^{k}\left(\begin{array}{c}
m \\
i
\end{array}\right)\left(\begin{array}{c}
m-i \\
k
\end{array}\right) x^{i+k}
$$

For $i=0,1, \ldots, m$. Let us denote $\psi(x)=\left[\mathrm{X}_{0, m}(x), \mathrm{X}_{1, m}(x), \ldots, \mathrm{X}_{m, m}(x)\right]$, then we have $\psi(x)=A T_{m}(x)$ where where $T_{m}(x)=\left[1, x, \ldots, x^{m}\right]$ and $I$ is $(m+1) \times(m+1)$ matrix defined by

$$
A=\left[\begin{array}{ccc}
(-1)^{0}\left(\begin{array}{c}
m \\
0
\end{array}\right) & \cdots & (-1)^{m-0}\left(\begin{array}{c}
m \\
0
\end{array}\right)\left(\begin{array}{c}
m-0 \\
m-0
\end{array}\right) \\
\vdots & \ddots & \vdots \\
0 & \cdots & (-1)^{m}\left(\begin{array}{l}
m \\
m
\end{array}\right)
\end{array}\right]
$$

Since $\operatorname{det}(A)=|A|=\prod_{i=0}^{m},\left(\begin{array}{c}m \\ i\end{array}\right) \neq 0$, it means that $A$ is an invertible matrix.

\subsection{Approximation of Functions}

A square integrable function $\mathrm{F}(\mathrm{x})$ in the interval $(0,1)$ may be approximated in terms of the basis of BPs $\psi^{T}(x)=\left[\mathrm{X}_{0, m}(x), \mathrm{X}_{1, m}(x), \ldots, \mathrm{X}_{m, m}(x)\right]$, (cf. [13]) as

$$
H^{T}\langle\psi(x), \psi(x)\rangle=\langle F(x), \psi(x)\rangle
$$

Where

$$
\langle F(x), \psi(x)\rangle=\int_{0}^{1} F(x) \psi(x)^{T} d x=\left[\left\langle F(x), \mathrm{X}_{0, m}(x)\right\rangle,\left\langle F(x), \mathrm{X}_{1, m}(x)\right\rangle \ldots,\left\langle F(x), \mathrm{X}_{m, m}(x)\right\rangle\right]
$$

And $\langle\psi(x), \psi(x)\rangle=Q$ is known as dual matrix of $\psi(x)$ and is given by the following relation

$$
Q=\langle\psi(x), \psi(x)\rangle=\int_{0}^{1} \psi(x) \psi(x)^{\mathrm{T}} \mathrm{dx}
$$

And the entries of $\mathrm{Q}$ are given by

$$
q_{i+1, j+1}=\int_{0}^{1} X_{i, m}(x), X_{j, m}(x) d x=\left(\begin{array}{c}
m \\
i
\end{array}\right)\left(\begin{array}{c}
m \\
j
\end{array}\right) \int_{0}^{1}(1-x)^{2 m-(i+j)} x^{i+j} d x=\frac{\left(\begin{array}{c}
m \\
i
\end{array}\right)\left(\begin{array}{c}
m \\
j
\end{array}\right)}{(2 m+1)\left(\begin{array}{c}
2 m \\
i+j
\end{array}\right)}
$$

Where $i, j=0,1, \ldots, m$, ultimately by (11) and (13), we get

$$
H^{T}=\left(\int_{0}^{1} f(x) \psi(x)^{T} d x\right) Q^{-1}
$$




\section{Operational Matrix for Fractional Integration Based on BPs}

The operational matrices of fractional Integration of the vector $\Psi(\mathrm{t})$ can be approximated as [14]:

$$
I_{t}^{\alpha} \psi(t) \cong F^{\alpha} \psi(t)
$$

Where $F^{\alpha}$ is the $(\mathrm{m}+1) \mathrm{x}(\mathrm{m}+1)$ is Riemann-Liuoville fractional matrix of integration for BPs, by the use of (6), we have

$$
I_{t}^{\alpha} \psi(t)=\frac{1}{\Gamma(\alpha)} \int_{0}^{t}(t-x)^{\alpha-1} \psi(x) d x=\frac{1}{\Gamma(\alpha)} t^{\alpha-1} * \psi(t)
$$

Where $*$ denotes the convolution product and by substituting $\psi(x)=A T_{m}(x)$ and from (4) we get

$$
\begin{aligned}
& \frac{1}{\Gamma(\alpha)} t^{\alpha-1} * \psi(t)=\frac{1}{\Gamma(\alpha)} t^{\alpha-1} *\left(A T_{m}(x)\right)=\frac{A}{\Gamma(\alpha)}\left(t^{\alpha-1} * T_{m}(x)\right) \\
& =\frac{A}{\Gamma(\alpha)}\left(t^{\alpha-1} * 1, t^{\alpha-1} * t, \ldots, t^{\alpha-1} * t^{m}\right)=A\left[I^{\alpha} 1, I^{\alpha} t, \ldots, I^{\alpha} t^{m}\right]^{T} \\
& =\frac{A}{\Gamma(\alpha)}\left[\frac{0 ! t^{\alpha}}{\Gamma(\alpha+1)}, \frac{1 ! t^{\alpha+1}}{\Gamma(\alpha+2)}, \ldots, \frac{m ! t^{\alpha+m}}{\Gamma(\alpha+m+1)}\right] T \\
& =A D \widetilde{T_{m}}
\end{aligned}
$$

Where $\mathrm{D}$ is an $(\mathrm{m}+1) \mathrm{x}(\mathrm{m}+1)$ matrix and $\widetilde{T_{m}}$ are given by

$D=\left[\begin{array}{ccc}\frac{0 !}{\Gamma(\alpha+1)} & 0 \cdots & 0 \\ \vdots & \ddots & \vdots \\ 0 & 0 \cdots & \frac{m !}{\Gamma(\alpha+m+1)}\end{array}\right]$ and $\widetilde{T_{m}}=\left[t^{\alpha}, t^{\alpha+1}, \ldots, t^{\alpha+m}\right]^{T}$,

Now we approximate $t^{\alpha+i}$ by $m+1$ terms of the BPs basis

$$
t^{\alpha+i} \approx E_{i}^{T} \psi(t)
$$

Therefore we have

$$
\begin{aligned}
E_{i}=Q^{-1}\left(\int_{0}^{1} t^{\alpha+i} \psi(t) d t\right) & =Q^{-1}\left[\int_{0}^{1} t^{\alpha+i} X_{0, m}(t) d t, \int_{0}^{1} t^{\alpha+i} X_{1, m}(t) d t, \ldots, \int_{0}^{1} t^{\alpha+i} X_{m, m}(t) d t\right]^{T} \\
& =Q^{-1} \widetilde{E}_{l}
\end{aligned}
$$

Where $\widetilde{E_{l}}=\left[\widetilde{E_{l, 0}}, \widetilde{E_{l, 1}}, \ldots, \widetilde{E_{l, m}}\right]$ and

$$
\widetilde{E_{l, m}}=\int_{0}^{1} t^{\alpha+i} X_{i, m}(t) d t=\frac{m ! \Gamma(i+j+\alpha+1)}{j ! \Gamma(i+j+\alpha+2)}, i, j=0,1, \ldots m
$$

Where $E$ is $(\mathrm{m}+1) \mathrm{x}(\mathrm{m}+1)$ matrix that has vector for $Q^{-1} \breve{E}_{l}$ ith column. Therefore, we can write 


$$
I_{t}^{\alpha} \psi(t)=A D\left[E_{0}^{T} \psi(t), E_{1}^{T} \psi(t), \ldots, E_{m}^{T} \psi(t)\right]^{T}=A D E^{T} \psi(t)
$$

Thus, we have

$$
I_{t}^{\alpha} \psi(t) \cong F^{\alpha} \psi(t)
$$

Where $F^{\alpha}=\mathrm{ADE}^{\mathrm{T}}$ is the fractional integration operational matrix.

\subsection{Operational Matrix for Product}

It is always necessary to assess the product of $\psi(t)$ and $\psi(t)^{T}$ which is called the product matrix forBPs basis. The operational matrices for the

$$
H^{T} \psi(t) \psi(t)^{T} \simeq \psi(t)^{T} \bar{H}
$$

Where $\bar{H}$ is an $(\mathrm{m}+1) \mathrm{x}(\mathrm{m}+1)$ matrix and we have

$$
\begin{gathered}
H^{T} \psi(t) \psi(t)^{T}=H^{T} \psi(t)\left(T_{m}{ }^{T}(x) A^{T}\right)=\left[H^{T} \psi(t), t H^{T} \psi(t), \ldots, t^{m} H^{T} \psi(t)\right] A^{T} \\
=\left[\sum_{i=0}^{m} c_{i} X_{i, m}(t), \sum_{i=0}^{m} c_{i} \mathrm{t} X_{i, m}(t), \ldots, \sum_{i=0}^{m} c_{i} t^{m} X_{i, m}(t)\right]
\end{gathered}
$$

Now, we approximate all functions $t^{k} \mathrm{X}_{i, m}(t)$ in terms of $\psi(t)$ for $\mathrm{i}, \mathrm{k}=0,1, \ldots \mathrm{m}$. By (10), we have

$$
t^{k} X_{i, m}(t) \simeq e_{k . i}^{T}(t) \psi(t)
$$

Where $e_{k, i}=\left[e_{k, i}^{0}, e^{1}{ }_{k, i}, \ldots, e^{m}{ }_{k, i},\right]$, then we obtain the components of vector $e_{k, i}$

$$
\begin{gathered}
e_{k, i}^{j} \simeq Q^{-1}\left(\int_{0}^{1} t^{k} X_{i, m}(t) \psi(t) d t\right) \\
=Q^{-1}\left[\int_{0}^{1} t^{k} X_{i, m}(t) X_{0, m}(t) d t, \int_{0}^{1} t^{k} X_{i, m}(t) X_{1, m}(t) d t, \ldots, \int_{0}^{1} t^{k} X_{i, m}(t) X_{m, m}(t) d t,\right]^{T} \\
=\frac{Q^{-1}}{2 m+k+1}\left[\frac{\left(\begin{array}{c}
m \\
0
\end{array}\right)}{\left(\begin{array}{c}
2 m+k \\
i+k
\end{array}\right)}, \frac{\left(\begin{array}{c}
m \\
1
\end{array}\right)}{\left(\begin{array}{c}
2 m+k \\
i+k+1
\end{array}\right)}, \ldots, \frac{\left(\begin{array}{c}
m \\
m
\end{array}\right)}{\left(\begin{array}{c}
2 m+k \\
i+k+m
\end{array}\right)}\right]^{T}, i, k= \\
0,1, \ldots, m .
\end{gathered}
$$

Thus

$$
\begin{aligned}
& \sum_{i=0}^{m} c_{i} \mathrm{t}^{\mathrm{k}} X_{i, m}(t) \simeq \sum_{i=0}^{m} c_{i}\left(\sum_{j=0}^{m} e_{k, i}^{j} X_{j, m}(t)\right)=\sum_{j=0}^{m} X_{j, m}(t)\left(\sum_{i=0}^{m} c_{i} e_{k, i}^{j}\right) \\
= & \psi(t)^{T}\left[\sum_{i=0}^{m} c_{i} e_{k, i}^{0}, \sum_{i=0}^{m} c_{i} e_{k, i}^{1}, \ldots, \sum_{i=0}^{m} c_{i} e_{k, i}^{m}\right]^{T}=\psi(t)^{T}\left[e_{k, 0}, e_{k, 1}, \ldots, e_{k, m}\right] H \\
= & \psi(t)^{T} V_{k+1} H,
\end{aligned}
$$


Where $V_{k+1}(k=0,1, \ldots, m)$ is an $(\mathrm{m}+1) \mathrm{x}(\mathrm{m}+1)$ matrix that has vectors $e_{k, i}$ given for each column. If we chose an $(\mathrm{m}+1) \mathrm{x}(\mathrm{m}+1)$ matrix $\bar{H}=\left[V_{1} c, V_{2} c, \ldots, V_{m+1} c\right]$, then from (25) and (28) we can write

$$
H^{T} \psi(t) \psi(t)^{T} \simeq \psi(t)^{T} \widetilde{H} A^{T}
$$

And therefore we obtain the operational matrix product $\bar{H}=\bar{H} A^{T}$.

Corrolary: If $y(t)=H^{T} \psi(t)$ consequently we can get the approximate function for $y^{k}(t)$, using BPs as follows

$$
y^{k}(t)=\psi(t)^{T} \bar{H}_{k}
$$

Where $\bar{H}_{k}=\bar{H}^{k-1} H$.

Proof. See the proof in [17].

\section{Scheme for the Numerical Solution of Fractional Order Logistic Equations using BPs}

In this section, we employ the operational matrices of BPs for solving the nonlinear FOLE (1). At first we expand fractional derivative in (1) by the Bernstein basis $\psi(t)$ as follows:

$$
D^{\alpha} y(t)=H^{T} \psi(t)
$$

Where

$$
H^{T}=\left[c_{0}, c_{1}, \ldots, c_{m}\right]
$$

And

$$
\psi^{T}(x)=\left[\mathrm{X}_{0, m}(x), \mathrm{X}_{1, m}(x), \ldots, \mathrm{X}_{m, m}(x)\right],
$$

are unknowns. Using initial condition (2) and (5), (23), we approximate $x(t)$ by

$$
x(t)=I_{x}^{\alpha} D_{x}^{\alpha} x(t)+x(0)=\left(H^{T} F^{\alpha}+d^{T}\right) \psi(t)=G^{T} \psi(t),
$$

Where $H^{T} F^{\alpha}+d^{T}=G^{T}$ and $F^{\alpha}$ is the fractional operational matrix of integration of order $\alpha$ and

$$
d^{T}=[b, b, \ldots, b]
$$

By substituting the approximations (31), (34) in (1) we have

$$
\begin{aligned}
H^{T} \psi(t) & =\beta G^{T} \psi(t)\left(I_{d}-G^{T} \psi(t)\right)=\beta\left(G^{T} \psi(t)-G^{T} \psi(t) \psi(t)^{T} G\right) \\
& =\beta\left(G^{T} \psi(t)-\psi(t)^{T} \bar{G} G\right)=\beta\left(G^{T}-G^{T} \bar{G}^{T}\right) \psi(t)
\end{aligned}
$$


Where $I_{d}$ is is the identity matrix. Thus from (36), we can get the following system by the use of the independent property of BPs functions,

$$
H^{T}-\beta\left(G^{T}-G^{T} \bar{G}^{T}\right)=0,
$$

from this system we can get $\mathrm{H}$ and hence we can approximate the solution of FOLE (1) i.e $\mathrm{x}(\mathrm{t})$, by using (34).

\section{Interpretation of Our Numerical Scheme}

Here we consider three examples of FOLE for the numerical solutions on the prescribed method, in order to check the accuracy of our scheme. We consider the solutions for different values of $\alpha$ and compare with the exact solutions in these examples.

Example 1 We consider a FOLE from [19]:

$$
\begin{gathered}
D^{\alpha} x(t)=\frac{1}{2} x(t)(1-x(t)), t>0,0<\alpha \leq 1, \\
x(0)=\frac{1}{2}
\end{gathered}
$$

for the numerical solutions for different values of $\alpha$ by our prescribed scheme. The solution is plotted in comparison with the exact solution

$$
x(t)=\frac{\operatorname{Exp}\left(\frac{t}{2}\right)}{1+\operatorname{Exp}\left(\frac{t}{2}\right)},
$$

Our approximations are too close to the exact solution in interval $[0,1]$ and beyond this interval gradual deviation is observed. We provide the solutions in interval $[0,2]$ and the error plot is given in interval $[0,1]$.

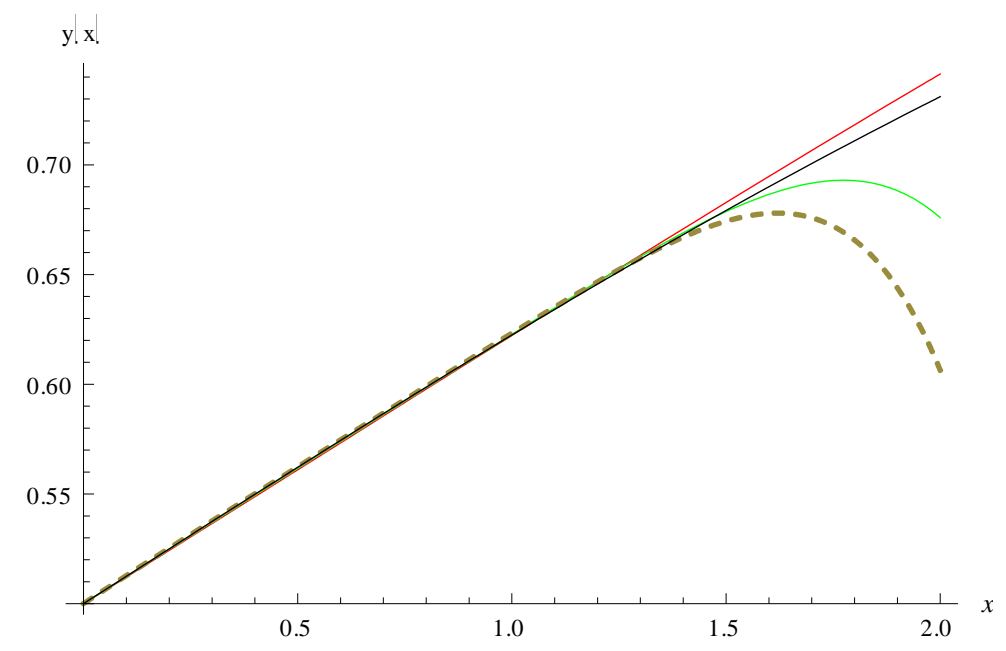

Figure 1: Approximation of FOLE in example 1, where the black line represents the exact solution, Red line is for $\alpha=1$, Green line is for $\alpha=.99$, Doted line is for $\alpha=.98$, and $x \in[0,2]$. 


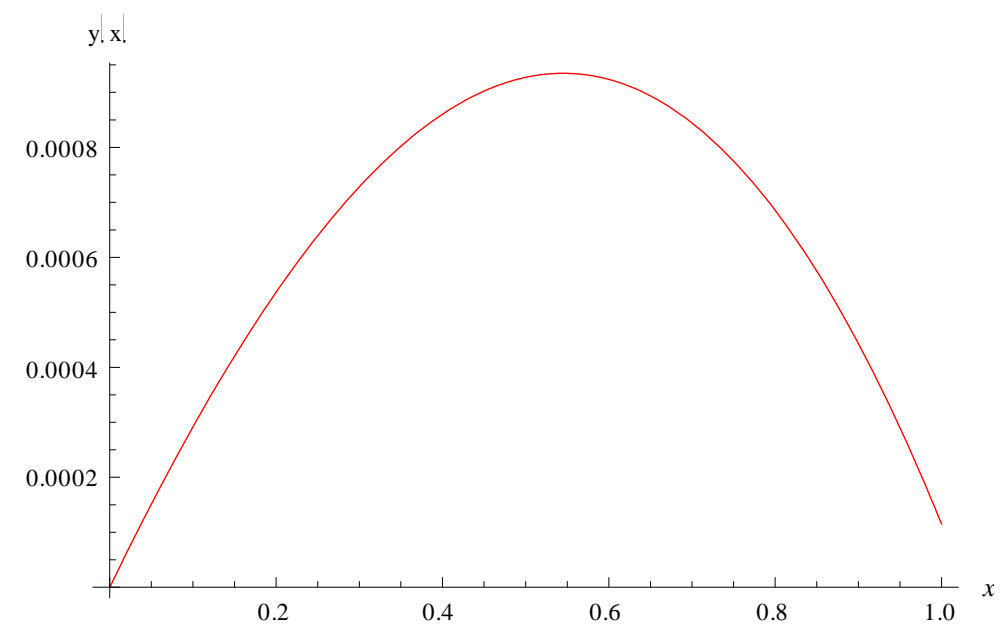

Figure 2: Error in our approximation for the FOLE in example 1 for $\alpha=1$ when $\mathrm{x} \in[0,1]$.

Example 2 We consider a FOLE from [19]:

$$
\begin{gathered}
D^{\alpha} x(t)=\frac{1}{4} x(t)(1-x(t)), \quad t>0, \quad 0<\alpha \leq 1, \\
x(0)=\frac{1}{3}
\end{gathered}
$$

for the numerical solutions for different values of $\alpha$ by our prescribed scheme. The approximate solutions are plotted in comparison with the exact solution

$$
x(t)=\frac{\operatorname{Exp}\left(\frac{t}{4}\right)}{2+\operatorname{Exp}\left(\frac{t}{4}\right)},
$$

which shows too better results in interval $[0,1]$ and beyond this interval little deviation is observed. We provide the solutions in interval $[0,2]$ and the error plot is given in interval $[0,1]$.

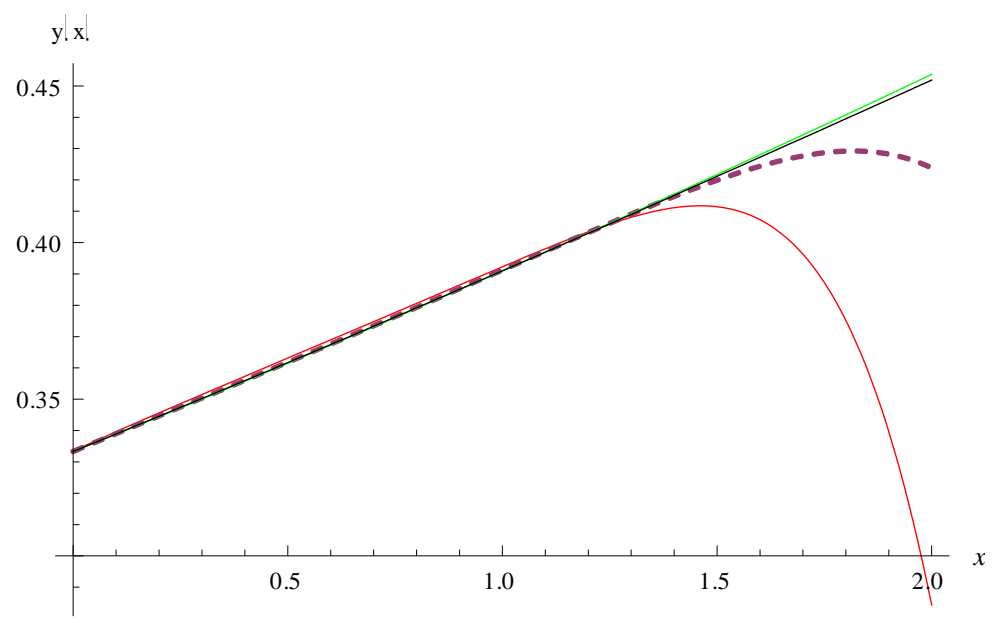

Figure 3: Approximation of FOLE in example 2 via BPs operational matrices. Here Black line=Exact solution, Green line is for $\alpha=1$, Doted line is for $\alpha=.99$, and the Red line is for $\alpha=.95$, and $\mathrm{x} \in[0,2]$. 


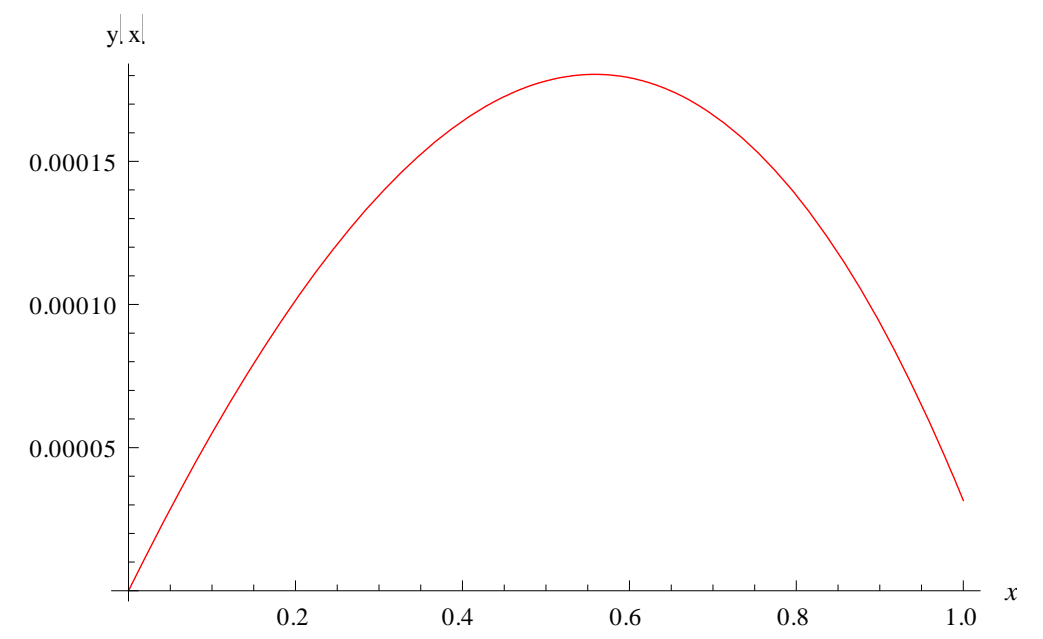

Figure 4: Error in our approximation of the FOLE in example 2 for $\alpha=1$ when $\mathrm{x} \in[0,1]$.

Example 3 We consider a FOLE:

$$
\begin{gathered}
D^{\alpha} x(t)=\frac{1}{3} x(t)(1-x(t)), \quad t>0, \quad 0<\alpha \leq 1 \\
x(0)=\frac{1}{3}
\end{gathered}
$$

for the numerical solutions for different values of $\alpha$ by our prescribed scheme. The solution is plotted in comparison with the exact solution

$$
x(t)=\frac{\operatorname{Exp}\left(\frac{t}{3}\right)}{2+\operatorname{Exp}\left(\frac{t}{3}\right)},
$$

The solutions obtained are tending close to the exact solution in interval $[0,1]$ when $\alpha$ approaches 1 and beyond this interval deviation is observed. We provide the solutions in interval $[0,2]$ and the error plot is given in $[0,1]$.

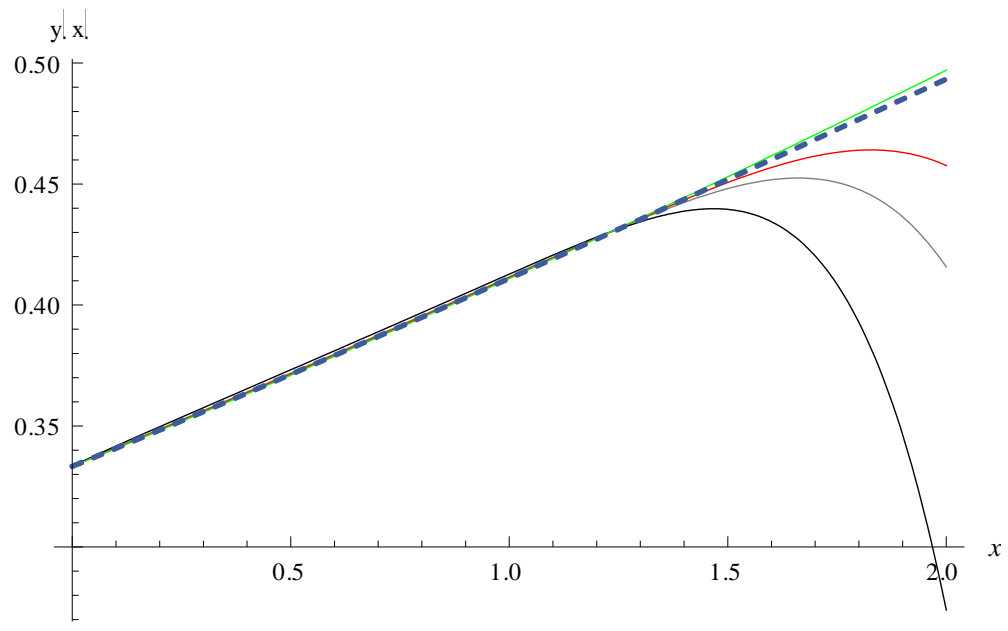

Figure 5: In this figure, we present comparison of our approximation with the exact solution in example 3 for different values of $\alpha$. Here the Dashed line represents exact solution at $\alpha=1$, where our 
approximations are: Green line is for $\alpha=1$, Red line is for $\alpha=.99$, Gray line is for $\alpha=.98$, Black line is for $\alpha=.95$, and $\mathrm{x} \in[0,2]$.

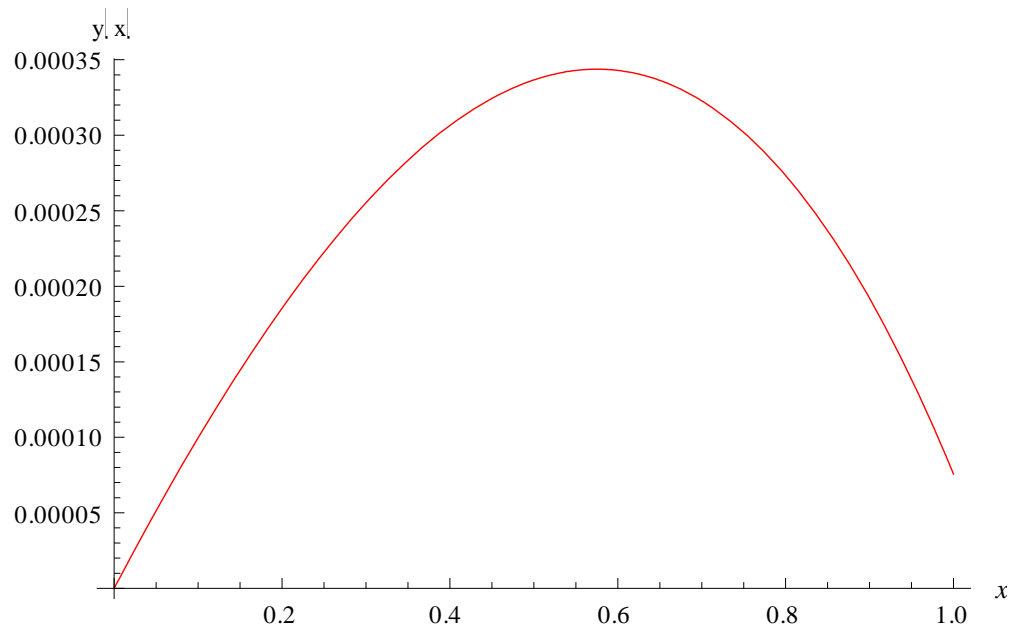

Figure 6: Error in our approximate solution of the FOLE in example 3 when $\alpha=1, x \in[0,1]$.

\section{Conclusion}

In this paper we have given a scheme for the numerical solution of FOLE based on the operational matrices of BPs for fractional order integration and multiplication. The method is too simple and highly efficient for the numerical solutions of the FOLE. The efficiency and simplicity of the scheme is given and justified by the three examples in the paper. In these examples, we see that better results are obtained for the values of $\alpha$ near by 1 , keeping the number of approximating terms $(m)$ the same. The simulations were carried out by Mathematica.

\section{References}

[1] R. Hilfer(Ed), "Application of fractional calculus in physics", World scientific publishing Co.Singapore, (2000).

[2] A. A. Kilbas, H. M. Srivastava, and J. J. Trujillo, "Theory and applications of fractional differential equations", Vol. 24, North-Holland Mathematics Studies, Amsterdame, (2006).

[3] K. S. Miller, B. Ross, "An introduction to the fractional calculus and fractional differential equations", Wiley, (1993).

[4] K. B. Oldhalm, J. Spainer, "The fractional calculus", Academic Press, New York, (1974).

[5] I. Podlubny, "Fractional differential equations", Academic Press, New York, (1999).

[6] R.L. Bagley, P.J. Torvik, "A theoretical basis for the application of fractional calculus to viscoelasticity”, J. Rheol. 27 (1983) 201-210.

[7] D. Baleanu, K. Diethelm, E. Scalas, J.J. Trujillo, "Fractional calculus models and numerical methods (Series on Complexity, Nonlinearity and Chaos)", World Scientific, (2012).

[8] M. Bhatti, P. Bracken, "Solutions of Differential Equations in a Bernstein Polynomial Basis", J. Comput. Appl. Math., 205 (2007) 272-280.

[9] T.S. Chow, "Fractional dynamics of interfaces between soft-nanoparticles and rough substrates", Phys. Lett. A, 342 (2005) 148-155. 
[10] E. H. Doha, A. H. Bhrawy, M. A. Saker, "Integrals of Bernstein polynomials: an application for the solution of high even-order differential equations", Appl. Math. Lett., 24 (2011) 559-565.

[11] H. Jafari, “An introduction to fractional differential equations", Mazandaran University Press, [Persian], (2013).

[12] H. Jafari, K. Sayevand, H. Tajadodi, D. Baleanu, "Homotopy analysis method for solving Abel differential equation of fractional order", Central European Journal of Physics, 11 (10) (2013) 1523-1527.

[13] K. Maleknejad, E. Hashemizadeh, B. Basirat, "Computational method based on Bernstein operational matrices for nonlinear Volterra-Fredholm-Hammerstein integral equations", Commun. Nonlinear Sci. Numer. Simul. 17 (1) (2012) 5261.

[14] D. Rostamy, M. Alipour, H. Jafari, D. Baleanu, "Solving multi-term orders fractional differential equations by operational matrices of BPs with convergence analysis", Romanian Reports in Physics, 65 (2)(2013) 334-349.

[15] S.A. Yousefi, M. Behroozifar, "Operational matrices of Bernstein polynomials and their applications", Int. J. Syst. Sci. Vol. 41, (6) (2010) 709-716.

[16] D. Baleanu, M. Alipour, H. Jafari, "The Bernstein operational matrices for solving the fractional quadratic Riccati differential equations with the Riemann-Liouville derivative", Abstract and Applied Analysis. Vol. (2013), Article ID 461970, 7 pages http://dx.doi.org/10.1155/2013/461970.

[17] M. Alipour, D. Rostamy, "Bernstein polynomials for solving Abels integral equation", The journal of mathematics and computer science. Vol .3 No.4 (2011) $403-412$.

[18] M. Alipour, D. Rostamy, "BPs operational matrices for solving time varying fractional optimal control Problems", Journal of mathematics and computer Science. 6 (2013) 292 - 304.

[19] S. Bhalekar, V. D. Gejji, "Solving fractional order Logistic equation using a new Iterative method", International Journal of Differential Equations, Vol. (2012), Article ID 975829, 12 pages, doi:10.1155/2012/975829.

[20] A. M. A. El-Sayed, A. E. M. El-Mesiry, H. A. A. El-Saka, "On the fractional-order logistic equation", Applied Mathematics Letters, vol. 20, no. 7 (2007), pp. 817823.

[21] V. Daftardar-Gejji, H. Jafari, "An iterative method for solving nonlinear functional equations", Journal of Mathematical Analysis and Applications, vol. 316, no. 2 (2006), pp. 753763.

[22] M. Akram, W. Shamaila, "Fixed point results in partial metric Spaces using generalized weak contractive conditions", J. Math. Computer Sci., 12 (2014), 85-98.

[23] Mostafa Allaoui, Abdel Rachid El Amrouss, Fouad Kissi, Anass Ourraoui, "Existence and multiplicity of solutions for a Robin problem”, J. Math. Computer Sci., 10 (2014), 163-172.

[24] M. M. Shamooshaky, P. Assari, H. Adibi, "The numerical solution of nonlinear FredholmHammerstein integral equations of the second kind utilizing Chebyshev wavelets", J. Math. Computer Sci., 10 (2014), 235-246.

[25] S. Akhavan, "Numerical solution of singular Fredholm integro differential equations of the second kind via Petrov Galerkin method by using Legendre multiwavelet", J. Math. Computer Sci., 9 (2014), 321-331. 